\title{
Isothermal Crystallization of Poly(tetrafluoroethylene). Effect of Holding Time in the Melt on Calorimetric Measurements
}

\author{
Ralph Jürgen LEHNERT ${ }^{\dagger}$ and Harald HIRSCHMANN*
}

Cavendish Laboratory, University of Cambridge, Cambridge CB3 OHE, UK

(Received July 5, 1996)

\author{
KEY WORDS Poly(tetrafluoroethylene)/ Isothermal Crystallization / Calorimetry / Holding Time in \\ Melt / Residual Order in Melt /
}

Crystalline poly(tetrafluoroethylene) (PTFE) exhibits three solid phases at atmospheric pressure with first order solid-solid transitions occurring at 292 and $303 \mathrm{~K}$. Depending on the experimental method and extrapolation used the equilibrium melting point lies between 600 and $620 \mathrm{~K} .^{1,2}$ As-polymerized (virgin) and meltcrystallized PTFE are known to be highly crystalline; fully amorphous polymer has not been observed. The microstructure of melt-crystallized bulk PTFE is unusual in that it consists predominantly of thick lamellae. Fibrillar and striated band-like morphologies have been observed under certain crystallization conditions for polymer of suitable molecular weight $t^{3,4}$ and incipient spherulitic structures with a large degree of imperfection were reported to occur at very slow cooling rates of the order of degrees Kelvin per hour. ${ }^{3}$ In a previous calorimetric study it was concluded from non-isothermal data obtained at constant cooling rate that PTFE crystallizes one-dimensionally from pre-existing nuclei which were presumed to be impurities. ${ }^{5}$ Neutron and X-ray diffraction, Raman spectroscopic, computational, and several other studies on molten PTFE all suggest that conformational, orientational, and local positional order persists after the polymer has been heated above the equilibrium melting point. ${ }^{6-12}$ The presumption that PTFE can retain some kind of order in the melt is in line with the outcome of a number of studies on the effect of melt holding time on the isothermal crystallization behaviour of polymers such as polyethylene, ${ }^{13}$ poly(ethylene oxide), ${ }^{13}$ poly(ethylene terephthalate), ${ }^{14-16}$ poly(ethere ether ketone), ${ }^{17,18}$ poly(phenylene sulfide), ${ }^{19,20}$ and polycarbonate. ${ }^{21}$ Many of the phenomena observed in these studies have been connected to self-nucleation by, for instance, Wunderlich in reference 22 which also provides quotations of similar studies on polymers not quoted above. The mechanism of self-nucleation, thereby microscopic remnants of polymer crystallites remain unmelted and can serve as nucleation sites on cooling below the melting temperature can be presumed to play a role also in the melt-crystallization of PTFE.

\section{EXPERIMENTAL}

As-polymerized commercial grade polymers were supplied in powder form by ICI, UK (Fluon G163)

\footnotetext{
$\uparrow$ To whom correspondence should be addressed.
}

* Present address: Merck KGaA, 64271 Darmstadt, Germany. and HOECHST AG, Germany (Hostaflon TF1750 and KU02). To determine the molecular weight according to Suwa et al.'s empirically derived relationship ${ }^{23}$ between the number-average molecular weight and the heat of crystallization about $7 \mathrm{mg}$ of polymer has been heated with a Perkin Elmer DSC-7 at a rate of $20 \mathrm{~K} \mathrm{~min}^{-1}$ from room temperature to $650 \mathrm{~K}$. The sample has then been held at this temperature for 5 minutes before cooling with $20 \mathrm{~K} \mathrm{~min}^{-1}$ back to room temperature. Heats of crystallization were obtained from the measured DSC exotherms and the molecular weight was estimated to be $44 \times 10^{5} \mathrm{~g} \mathrm{~mol}^{-1}, 22 \times 10^{5} \mathrm{~g} \mathrm{~mol}^{-1}$, and $2 \times 10^{5} \mathrm{~g} \mathrm{~mol}^{-1}$ for G163, TF1750, and KU02, respectively. The peak melting temperatures $T_{\mathrm{m}}$ were obtained using the same calorimeter at a heating rate of $20 \mathrm{~K} \mathrm{~min}^{-1}$. Values for $T_{\mathrm{m}}$ were found to be 619,617 , and $607 \mathrm{~K}$, in the order of falling molecular weight. The last traces of the melting endotherms vanished some 5 to $7 \mathrm{~K}$ above $T_{\mathrm{m}}$.

Isothermal crystallization experiments were performed on the same instrument in a dry nitrogen gas flow. Powdered polymer with a weight of $13.7 \pm 0.5 \mathrm{mg}$ was sealed in aluminium pans and heated at a rate of $20 \mathrm{~K} \mathrm{~min}^{-1}$ from room temperature to $650 \mathrm{~K}$. This temperature which is typical for PTFE sintering processes was then held for different times prior to cooling at a rate of $100 \mathrm{~K} \mathrm{~min}^{-1}$ to the crystallization temperature, $T_{\mathrm{c}}$. All holding times were sufficiently long to allow the heat flow to equilibrate at a constant rate, indicating that melting was completed before the sample was cooled. At $T_{\mathrm{c}}$ the heat flow through the sample was recorded from the end of the transient instrument response (approximately 25 seconds after $T_{\mathrm{c}}$ was reached) until a constant signal representing negligible thermal activity of the sample was measured. Finally, the isothermal part of a crystallization experiment was ended by heating the sample at $20 \mathrm{~K} \mathrm{~min}^{-1}$ to determine the heat of fusion. Thermogravimetric analysis confirmed that polymer degradation did not occur during temperature cycles with holding times of up to 10 hours.

\section{RESULTS AND DISCUSSION}

Preliminary experiments were conducted with the aim of finding a temperature domain suitable for the observation of isothermal melt-crystallization. The previous study by $\mathrm{Ozawa}^{5}$ on the non-isothermal crystallization of PTFE pointed to a temperature interval ranging between 580 and $590 \mathrm{~K}$. In the present work, 
meaningful heat flow curves could be recorded in the narrow range between 590 and $595 \mathrm{~K}$. Below $590 \mathrm{~K}$ crystallization had already been completed within the approximately 60 seconds that elapsed from the start of the temperature ramp down until the instrument transient had decayed away. Above $595 \mathrm{~K}$ samples remained molten after more than 1 hour, as indicated by the absence of a melting endotherm on reheating. The effect of holding time at $650 \mathrm{~K}$ on the heat flow curves of grade KU02 was then investigated at $T_{\mathrm{c}}=594 \mathrm{~K}$. The latter temperature was chosen to minimise interference of the instrument transient with the crystallization exotherm and to ensure that crystallization was completed after typically 10 to 15 minutes. Figure 1 shows heat flow curves which were recorded for holding times varied between 30 seconds and 64 minutes. The plot clearly reveals that the overall crystallization rate falls with increasing holding time. All samples were kept for 20 minutes at $594 \mathrm{~K}$ and crystallization was completed before they were heated again. The heat of fusion $\Delta h_{\mathrm{f}}$ was found to lie between 42.9 and $44.4 \mathrm{~J} \mathrm{~g}^{-1}$ with the values showing no trend, indicating that the final degree of crystallization by DSC was independent of the holding time within error limits. Heats of crystallization, $\Delta h_{\mathrm{c}}$ were determined by numerical integration from the measured heat flow curves. The missing initial parts were extrapolated assuming a simple cubic time dependence. Values for $\Delta h_{\mathrm{c}}$ typically range from 22 to $28 \mathrm{~J} \mathrm{~g}^{-1}$ and show a tendency to decrease for longer holding times. With these values the ratio $\Delta h_{\mathrm{c}} / \Delta h_{\mathrm{f}}$ can be calculated to lie between about 0.50 and 0.65 suggesting that another crystallization process exists. Whether this assumed second process takes place during the cooling or reheating stage or both could not be established but, clearly, signs for the occurrence of a distinct exothermic process other than the one already described were not observed. The values for $\Delta h_{\mathrm{c}} / \Delta h_{\mathrm{f}}$ are in good agreement with the investigation by Ozawa who measured values between 0.3 and 0.5 and explained the discrepancy of these figures from unity by secondary crystallization. Supporting evidence for this proposal is provided by an electron microscopic (EM) study by Melillo and Wunderlich $^{3}$ where it was found that PTFE lamellae increase their thickness by about $40 \%$ within 40 minutes on annealing at $589 \mathrm{~K}$. They attributed the observed thickness changes to solid state thickening following the solidification step during melt-crystallization. Another EM study on bulk PTFE by Bassett and Davitt ${ }^{24}$ found clear evidence of substantial lamella thickening at $594 \mathrm{~K}$ but failed to observe any changes at $589 \mathrm{~K}$ after times as long as 18 hours. Undoubtedly, even a slightly different temperature calibration, a differing molecular weight or polydispersity can markedly affect the outcome of studies of this kind. In view of this and since the molecular weight distribution remained unspecified in the papers quoted it can only be stated that substantial lamella thickening can occur in the temperature region relevant here and that the accompanying change in enthalpy can be expected to contribute significantly to the measured difference between $\Delta h_{\mathrm{c}}$ and $\Delta h_{\mathrm{f}}$.

To investigate the effect of different molecular weight on the calorimetric measurements, samples of grade G163 and TF1750 were subjected to the same type of

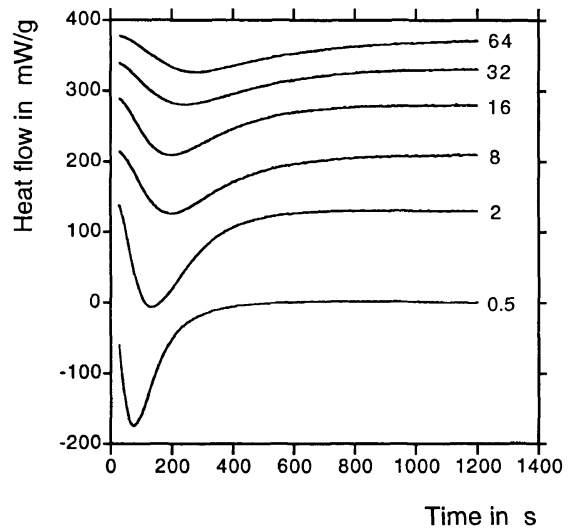

Figure 1. Heat flow curves during isothermal crystallization of PTFE grade KU02 at $594 \mathrm{~K}$. Plot is annotated with holding times in minutes at $650 \mathrm{~K}$. Curves other than " 0.5 " are shifted along the ordinate for clarity.

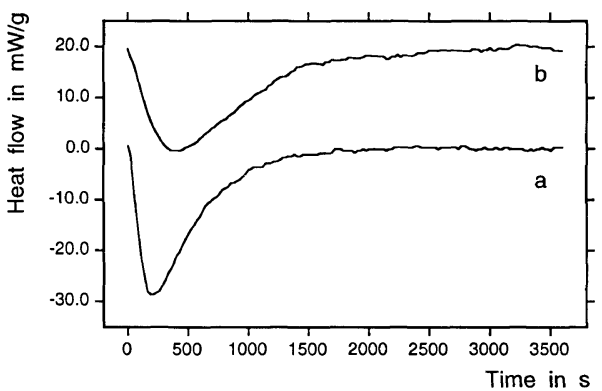

Figure 2. Heat flow curves during isothermal crystallization of PTFE grades $\mathrm{G} 163$ (a) and TF1750 (b) at $594 \mathrm{~K}$. Holding time for both grades at $650 \mathrm{~K}$ was 600 minutes. Curve (b) is shifted along the ordinate for clarity. Note different time scale compared to Figure 1.

heating-cooling cycle. For both grades crystallization was found to be completed to an overwhelming extent during cooling for holding times shorter than 3 hours. Exothermic peaks and heat flow curves similar to the ones shown in Figure 1 could only be observed at 10 hours holding time. Representative heat flow curves are depicted in Figure 2. The heats of fusion after 10 hours in the molten state and 1 hour at $T_{\mathrm{c}}=594 \mathrm{~K}$ were determined to be 41.9 and $43.3 \mathrm{Jg}^{-1}$ for grade $\mathrm{G} 163$ and TF1750, respectively. These values and the corresponding ratios $\Delta h_{\mathrm{c}} / \Delta h_{\mathrm{f}}$ which were calculated to be 0.40 and 0.49 compare favourably with the findings on KU02. Obviously, the higher molecular weight polymers are affected by a longer holding time in a way similar to grade KU02 with the difference being, that at the same holding time grade KU02 crystallizes more slowly than the other two grades. Whether the maximum holding times of, respectively, 64 and 600 minutes employed in this study were sufficient for the respective polymer melts to reach an equilibrium state whose crystallization behaviour would remain unchanged on further increasing the time spent at $650 \mathrm{~K}$ was not investigated. To gain information about the nucleation mechanism the experiments were repeated incorporating different amounts of dried talc (hydrous magnesium silicate, particle size $\leq 10 \mu \mathrm{m})$ as a nucleating agent. The crystallization behaviour was found to be unchanged despite the attempt to induce regular heterogeneous nucleation which is in accord with findings by Bassett and Davitt ${ }^{24}$ 
who also unsuccessfully tried to induce heterogeneous nucleation on foreign surfaces.

A tentative explanation for the observations can be given on the assumption that melt-crystallization in PTFE is facilitated by regions with some kind of (admittedly not further specified) residual order. It does not seem unreasonable to assume that the ease with which crystallization takes place is directly related to the amount of order retained in the melt. The degree of residual order can be expected to decrease as time proceeds and to do so more quickly as the molecular mobility increases and, accordingly, as the melt viscosity falls. This view is corroborated by the observations described in references 6 to 12 all of which hint at residual order in the melt. In addition the extremely high melt viscosity of PTFE in comparison with other common polymers makes it plausible that effects related to residual order are more likely to occur in molten PTFE than in other polymer melts. Since the melt viscosity of sufficiently long polymers (the entanglement threshold of about 500 monomer units corresponds to a molecular weight of $5 \times 10^{4}$ $\mathrm{g} \mathrm{mol}^{-1}$ for PTFE) scales with the molecular weight, $M$ as $M^{3.4}$ polymer KU02 can be expected to be between three and four orders of magnitude less viscous than the other two grades investigated here. This certainly justifies the proposal that residual order decays more rapidly in the melt of lower molecular weight PTFE. Consequently the overall crystallization rate would for the same melt residence time be anticipated to increase as the molecular weight is raised, which was indeed observed in this study. Also in line with this tentative explanation is the observation that for the same polymer grade longer holding times yield slower overall crystallization rates; residual order in the melt diminishes with time, necessitating cooperative molecular motion to a larger extent to form crystalline lamellae which, in turn, slows down crystallization. It appears probable that the residual order in the melt invoked to interpret the data presented in this note can be identified with self-nucleation seeds. Insufficient melt holding times and/or too high molecular weights of the PTFE grades investigated in previous studies have presumably been the reason that isothermal melt-crystallization of PTFE was widely believed to proceed too rapidly to be observable using calorimetric techniques.

\section{REFERENCES}

1. B. Wunderlich, "Macromolecular Physics," Vol. 3, Crystal Melting, Academic Press, New York, N.Y., 1980, p 48.

2. H. W. Starkweather, Macromolecules, 19, 1131 (1986).

3. L. Melillo and B. Wunderlich, Kolloid Z.-Z. Polym., 250, 417 (1972).

4. T. Suwa, T. Seguchi, M. Takehisa, and S. Machi, J. Polym. Sci., Part B, Polym. Phys., 13, 2183 (1975).

5. T. Ozawa, Bull. Chem. Soc. Jpn., 57, 952 (1984).

6. B. Rosi-Schwartz and G. R. Mitchell, Polymer, 35, 3139 (1994).

7. R. Lovell, G. R. Mitchell, and A. H. Windle, Farady Discus. R. Soc. Chem., 68, 46 (1980).

8. A. E. Tonelli, Polymer, 17, 695 (1976).

9. Y. P. Khanna, G. Chomyn, R. Kumar, N. S. Murthy, K. P. O'Brian, and A. C. Reimschuessel, Macromolecules, 23, 2488 (1990).

10. S. Sherratt, "Polytetrafluoroethylene," in "Kirk-Othmer Encyclopedia of Chemical Technology," 2nd ed, Interscience Publishers Inc., New York, N.Y., 1966, p 818f.

11. H. W. Starkweather, J. Polym. Sci., Part B, Polym. Phys., 23, 1177 (1985).

12. R. J. Lehnert, P. J. Hendra, N. Everall, and N. J. Clayden, Polymer, in press.

13. G. Vidotto, D. Levy, and A. J. Kovacs, Kolloid Z.-Z. Polym., 230, 289 (1969).

14. S. P. Kim and S. C. Kim, Polym. Eng. Sci., 33, 83 (1993).

15. A. Keller, G. R. Lester, and C. B. Morgan, Phil. Trans. R. Soc. London A247, 1 (1954).

16. F. D. Hartley, F. W. Lord, and C. B. Morgan, Phil. Trans. R. Soc. London A247, 23 (1954)

17. Y. Deslandes, M. Day, N. Sabir, and T. Suprunchuk, Polym. Compos., 10, 360 (1989).

18. Y. Lee and R. S. Porter, Macromolecules, 21, 2770 (1988).

19. N. A. Mehl and L. Rebenfeld, Polym. Eng. Sci., 32, 1451 (1992).

20. D. R. Budgell and M. Day, Polym. Eng. Sci., 31, 1271 (1991).

21. F. Gallez, R. Legras, and J. P. Mercier, J. Polym. Sci., Part B, 14, 1367 (1976).

22. B. Wunderlich, "Macromolecular Physics," Vol. 2, Crystal Nucleation, Growth, Annealing, Academic Press, New York, N.Y., 1976, p 52f.

23. T. Suwa, M. Takehisa, and S. Machi, J. Appl. Polym. Sci., 17, 3253 (1973).

24. D. C. Bassett and R. Davitt, Polymer, 15, 721 (1974). 